\title{
Mission archéologique franco-égyptienne de Tell el-Herr (2020)
}

Premier bilan de l'étude du matériel archéologique

Catherine Defernez, Nathalie Favry, Aline Banaszak et Sépideh Qaheri

\section{CpenEdition}

Journals

Édition électronique

URL : https://journals.openedition.org/baefe/2870

DOI : $10.4000 /$ baefe.2870

ISSN : 2732-687X

Éditeur

ResEFE

Référence électronique

Catherine Defernez, Nathalie Favry, Aline Banaszak et Sépideh Qaheri, « Mission archéologique francoégyptienne de Tell el-Herr (2020) » [notice archéologique], Bulletin archéologique des Écoles françaises à l'étranger [En ligne], Égypte, mis en ligne le 30 mai 2021, consulté le 27 juillet 2021. URL : http:// journals.openedition.org/baefe/2870 ; DOI : https://doi.org/10.4000/baefe.2870

Ce document a été généré automatiquement le 27 juillet 2021.

\section{(i) 8}

Le Bulletin archéologique des Écoles françaises à l'étranger est mise à disposition selon les termes de la Licence Creative Commons Attribution - Pas d'Utilisation Commerciale - Pas de Modification 4.0 International. 


\section{Mission archéologique franco- égyptienne de Tell el-Herr (2020)}

Premier bilan de l'étude du matériel archéologique

Catherine Defernez, Nathalie Favry, Aline Banaszak et Sépideh Qaheri

\section{NOTE DE L'AUTEUR}

Année de la campagne : 2020

Autorité nationale présente : Le Ministère du Tourisme et des Antiquités (MoTA) était représenté par Metwali Saleh, Qutub Mustafa Qutub et Nader Galal

Numéro et intitulé de l'opération de terrain : 19117 - Mission archéologique francoégyptienne de Tell el-Herr

Composition de l'équipe de terrain : L'équipe de terrain était composée de Catherine Defernez (CNRS, UMR 8167), Nathalie Favry (égyptologue, Sorbonne Université, UMR 8167), Pascale Ballet (université Paris Nanterre, UMR 7041 ArScan/ESPRI), Sépideh Qaheri (égyptologue, Collège de France), Séverine Marchi (archéologue, CNRS, UMR 8167), Aline Banaszak (doctorante, université Lille 3, CNRS, UMR 8164 HALMA), Anne-Laure Daubisse (doctorante, Sorbonne Université), Elsayed Abd el-Alim (directeur de l'inspectorat de Qantara, MoTA).

Partenariats institutionnels et organismes financeurs :

- Ministère de l'Europe et des Affaires étrangères (MEAE)

- CNRS, UMR 8167 Orient \& Méditerranée, équipe Mondes pharaoniques

- Sorbonne Université (faculté des lettres)

Donnée scientifiques produites :

http://www.ifao.egnet.net/archeologie/tell-el-herr/ 


\section{Campagne automne 2020}

1 La situation sanitaire n'a pas permis, à l'automne 2020, la tenue de la cinquième campagne d'étude de la mission de Tell el-Herr ${ }^{1}$, dans la ville de San el-Haggar (Sharquiya), où est aujourd'hui conservée une part importante de la documentation archéologique amassée au cours des vingt-cinq années d'activités sur le terrain (fig. 1). Les membres de l'équipe franco-égyptienne ont toutefois poursuivi activement leurs travaux de valorisation du site - et de la région - en concentrant leurs efforts à la préparation et à la rédaction des publications de synthèse d'une phase importante de l'histoire du tell, associée aux niveaux de la fin du $v^{e}$ et du Ive $s$. av. J.- $C^{2}$. Plusieurs d'entre eux, en charge des dossiers en cours (voir infra), ont participé, en 2020, à la rédaction d'un volume collectif entièrement dévolu au mobilier archéologique de Tell el-Herr et des sites avoisinants; les collaborateurs de la mission intervenant sur d'autres sites majeurs de la région (Tell Heboua I-II, Tell el-Farama/Péluse, Tell elMakhzan (fig. 2) y ont également contribué. Les dix articles réunis dans cet ouvrage (Tell el-Herr. Varia : études du mobilier archéologique) sont en cours de révision et seront publiés prochainement dans un supplément de la revue numérique de la Sorbonne ( $\mathrm{NeHet}$ ), sous la tutelle conjointe de la Sorbonne Université et de l'UMR 8167.

Dans le cadre des publications programmées, les études des diverses catégories d'objets identifiées sur le site ont nettement progressé. Les résultats exposés ici (coroplathie, céramique, bijoux, lithique) démontrent la richesse de la documentation de ce site clé du littoral nord-sinaïtique, qui demeure toujours, à ce jour, l'un des principaux acteurs de la culture matérielle perse en Égypte.

Fig. 1. Vue du tell, juillet 2019 (E. Abd el-Alim).

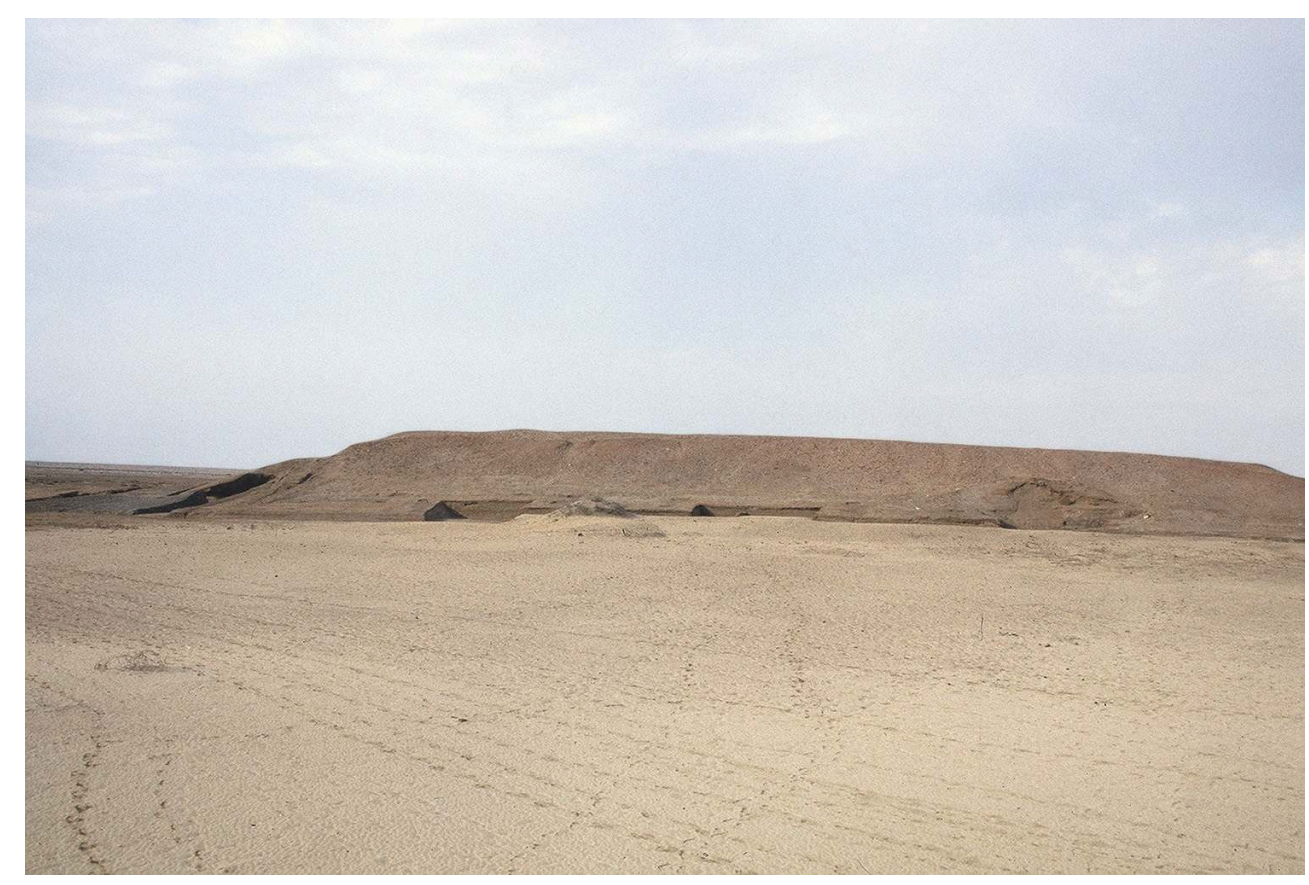

(C) Ifao. 19117_2020_NDMPF_001 
Fig. 2. Carte des principaux sites de l'angle nord-ouest du Sinaï (N. Favry).

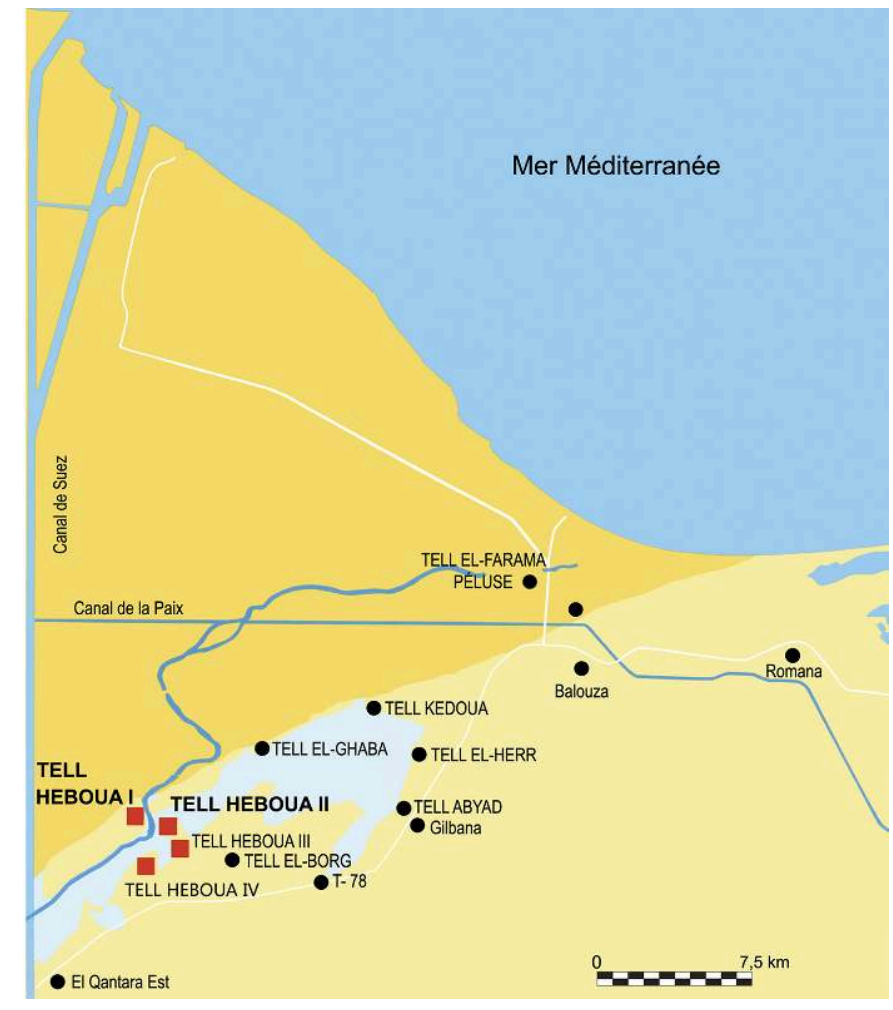

(C) Mission franco-égyptienne de Tell el-Herr/Ifao. 19117_2020_NDMCN_001

\section{2. Étude céramologique}

Catherine Defernez

\subsection{Compléments d'étude de la documentation des quartiers ouest, nord-ouest et sud-est du tell}

3 Selon les priorités définies dans le cadre de la mission - la préparation des prochains ouvrages collectifs -, l'étude s'est concentrée sur le matériel extrait des quartiers d'habitations nord-ouest et ouest du tell, contemporains de l'édifice palatial récemment publié3. Les recherches sur les catégories de vaisselles fines communes atypiques, peu représentées dans les répertoires classiques habituels de la fin du $\mathrm{I}^{\mathrm{er}}$ millénaire av.J.-C., ont néanmoins été privilégiées. Vases à liquides, de service (cruches, bouteilles, flacons et pichets), amphores de table, vaisselles de «table » ou d'apparat (coupelles, bols et plats à marli, de tradition hellénique, phénicienne et assyrienne), ou à usage spécifique, vouées à l'éclairage ou à des pratiques cultuelles (lampes diverses, encensoirs ou thymiateria), les céramiques identifiées offrent une variété infinie de formes et de modules - notamment dans la gamme des vases dits de service. D'origine égyptienne mais non locale ${ }^{4}$, leur fabrique - majoritairement à base de marnes, finement décantée et épurée - traduit une finesse d'exécution et de finition comparables à celles de leurs pendants égéens ou proche-orientaux, également notables sur le site ${ }^{5}$. 
imitations de vaisselles d'apparat en pierre, en métal ou tout autre matériau noble, ont fait l'objet de compléments d'étude, dans l'optique d'une prochaine rencontre scientifique à l'Ifao (voir infra). Des données intéressantes sont apparues, en particulier une marque estampée d'un motif de griffon ailé sur la panse d'un bol de tradition assyrienne ${ }^{6}$. Les protomes d'oiseau griffon, avec enroulement autour du cou et bouton sur le front, ne sont pas rares à l'époque achéménide : le mobilier d'apparat en métal, en pierre ou en bois, en offre diverses attestations. Cependant, sur les timbres principalement amphoriques - jusqu'ici connus, ce motif n'est pas très répandu et ne semble pas avoir été employé, en dehors de la cité d'Abdère. À ce jour, aucun parallèle précis n'a encore été identifié dans les corpus abondants que constituent ces documents.

5 La chronologie des phases récentes du tell (phases V-IV, fin $V^{\mathrm{e}}-\mathrm{IV} \mathrm{e}^{\mathrm{e}} \mathrm{s}$. av. J.-C.) s'est aussi affinée grâce à l'apport de l'étude de fragments d'origine grecque, souvent insignifiants au moment de leur découverte, mais riche d'enseignements à tous égards, une fois leur profil défini. Des tessons de vases attiques à vernis noir et à figures rouges ont procuré de nouveaux jalons chronologiques pour les ensembles étudiés lors de la dernière campagne (îlots VIII-IX, N99, N138/N139, 0153, 0154)7 ; même si, à la différence de bien d'autres contextes, les témoignages sont rares $^{8}$, ils demeurent éloquents sur le plan chronologique. De beaux exemples de lécythes aryballisques à décor de godrons et de larges palmettes, de olpè (pseudo-attiques), de plats et de coupes à vernis noir et décor estampé de palmettes accostées de rinceaux complètent le vaste répertoire déjà connu (fig. 3).

De manière générale, les trouvailles attiques sont plus fréquentes dans les niveaux perses précoces du tell, attribuables aux phases VB et VI ( $\mathrm{V}^{\mathrm{e}} \mathrm{s}$. av. J.-C.). Les ensembles étudiés lors de la dernière saison provenant de l'esplanade ou de la cour 088, aménagée sur le flanc nord-ouest du site, ont livré plusieurs fragments de vases à boire et de lécythes, dont l'identification typologique a pu être assurée, malgré leur forme résiduelle. De toute évidence, ces tessons documentent plusieurs des catégories constantes du répertoire achéménide : cercle de Beldam, groupes de la Saint-Valentin, des owl-skyphoi, des echinus-bowls, des skyphoi de type A et des convex-concave cups, bien attestés dans les corpus de l'Agora d'Athènes. L'étude exhaustive des assemblages extraits de la fouille de divers sondages pratiqués à l'opposé du tell', examinés l'an dernier, offre un répertoire analogue. Aux formes mentionnées viennent s'ajouter, cependant, d'autres lécythes à motifs figurés et vernis noir (fig. 4-5), des lampes, des coupes à anses au fond estampé de palmettes (fig. 3) et des fragments de coupes attribuables au cercle du peintre de Haimon ${ }^{10}$. 
Fig. 3. Coupe attique à décor estampé de palmettes (inv. P07/177, N. Favry).
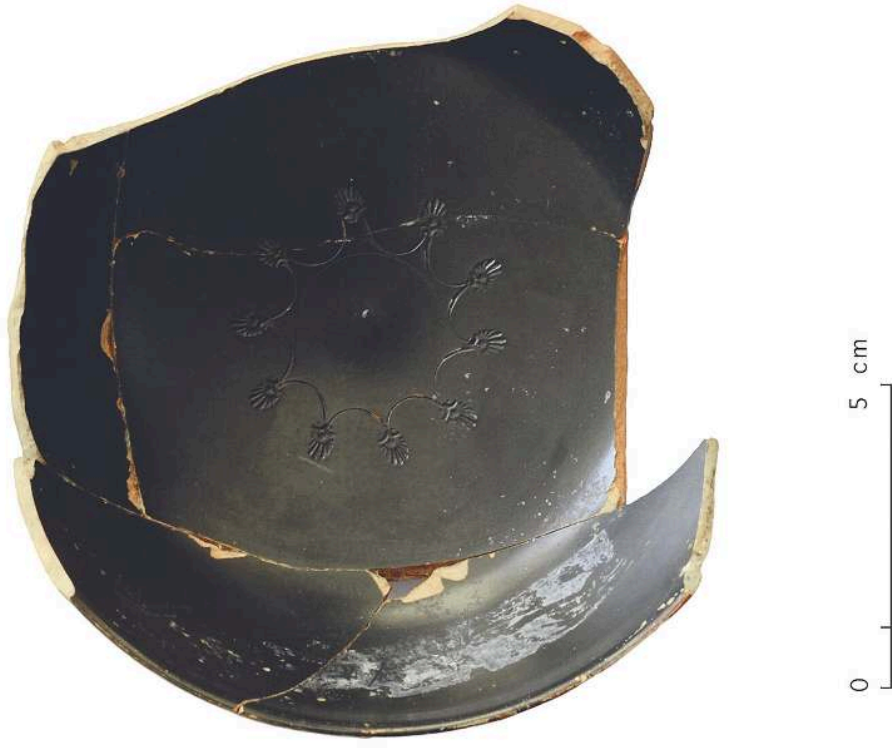

(C) Ifao. 19117_2020_NDMPM_001

Fig. 4. Fragment d'un lécythe aryballisque, buste de sphinge (inv. P08/109, P. Le Guilloux).
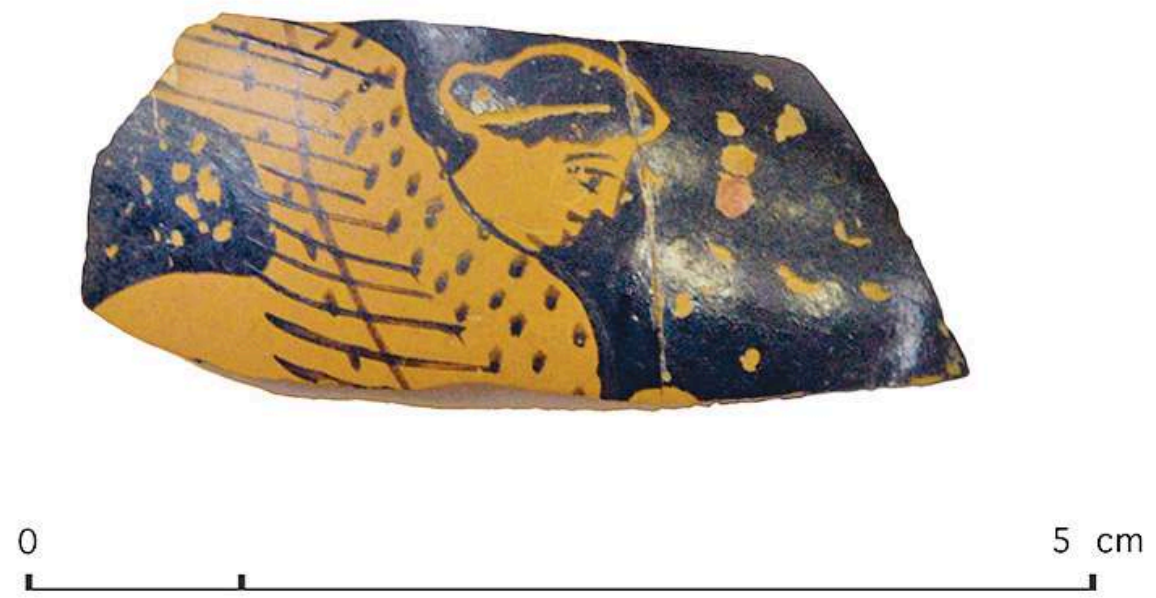
Fig. 5. Fragment d'un lécythe aryballisque, servante vêtue d'un chiton - secteur nord-est, phase VA (inv. P87/278, J.-F. Gout).
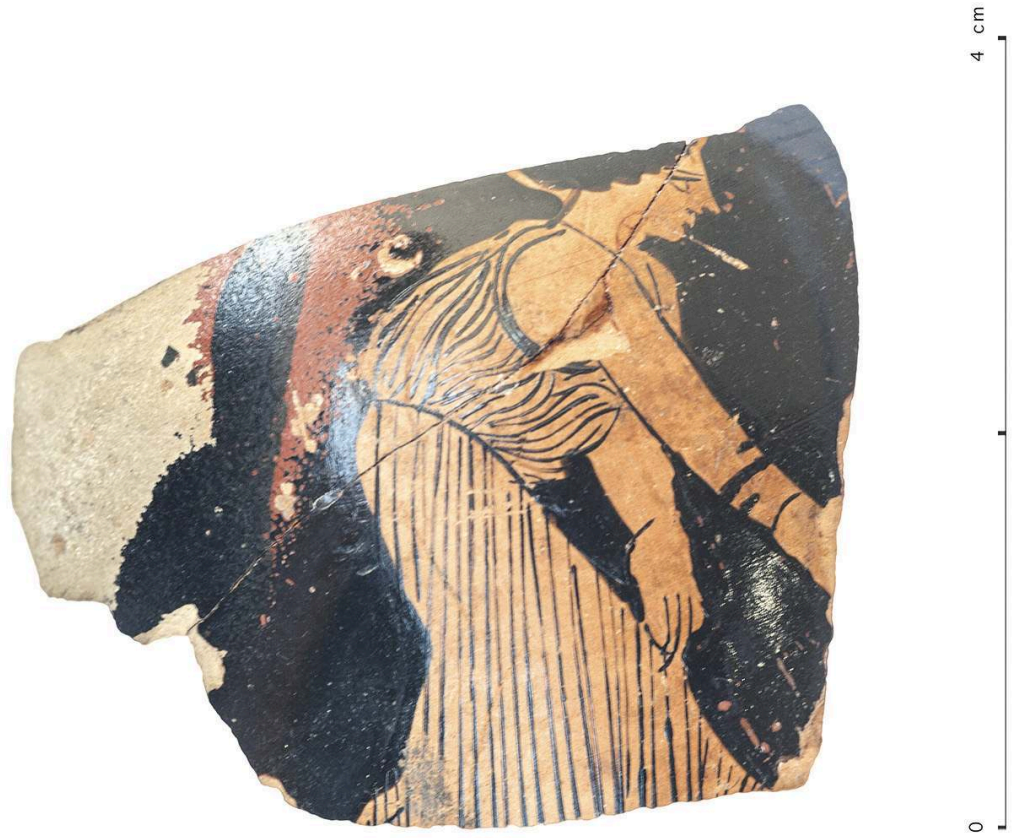

\section{@ Ifao. 19117_2020_NDMPM_003}

Dans la catégorie de la vaisselle fine attique, l'avancée des recherches a en outre permis l'attribution de documents plus rares à des artistes renommés, ayant joui d'une activité importante dans la deuxième moitié du $\mathrm{v}^{\mathrm{e}} \mathrm{s}$. av. J.-C. De beaux fragments de cratères en cloche, à volutes, et peut être en calice, mettant en scène des personnages dionysiaques (ménades et satyres) ou des cérémonies festives (symposia), peuvent dorénavant être assignés aux peintres de Cléophon, de Cassel/Clio et de Méléagre, ou à leur entourage ${ }^{11}$; à l'exception de Naucratis et de Memphis ${ }^{12}$, les œuvres de ces artistes restent peu documentées dans la satrapie égyptienne. De même, plusieurs fragments de panses d'un cratère campaniforme sur lesquels sont dépeints des hoplites semblent témoigner de la diffusion des œuvres du groupe de Polygnotos. D'autres tessons appartenant à des coupes-skyphoi, des askoi, des lécythes et des amphores de table démontrent également, à travers l'iconographie de leur décor, la richesse du répertoire attique, actuellement mis au jour dans la forteresse perse.

8 Comme il a déjà été indiqué, les importations de vaisselles fines ne se limitent pas à la seule sphère grecque. D'autres types de vases d'une qualité aussi remarquable que celle des productions athéniennes sont aussi bien attestées et viennent apporter leur lot d'informations. Des vases linéaires peints ${ }^{13}$ (fig. 6), bien représentés au Levant-Nord, ont récemment été attribués à un centre producteur de Cilicie, localisé aux alentours de Kelenderis, d'après les résultats des données archéométriques fournies par le matériel similaire $^{14}$. 
Fig. 6. Bouteille à décor linéaire peint - secteur est, phase VA (inv. P10/118, N. Favry).

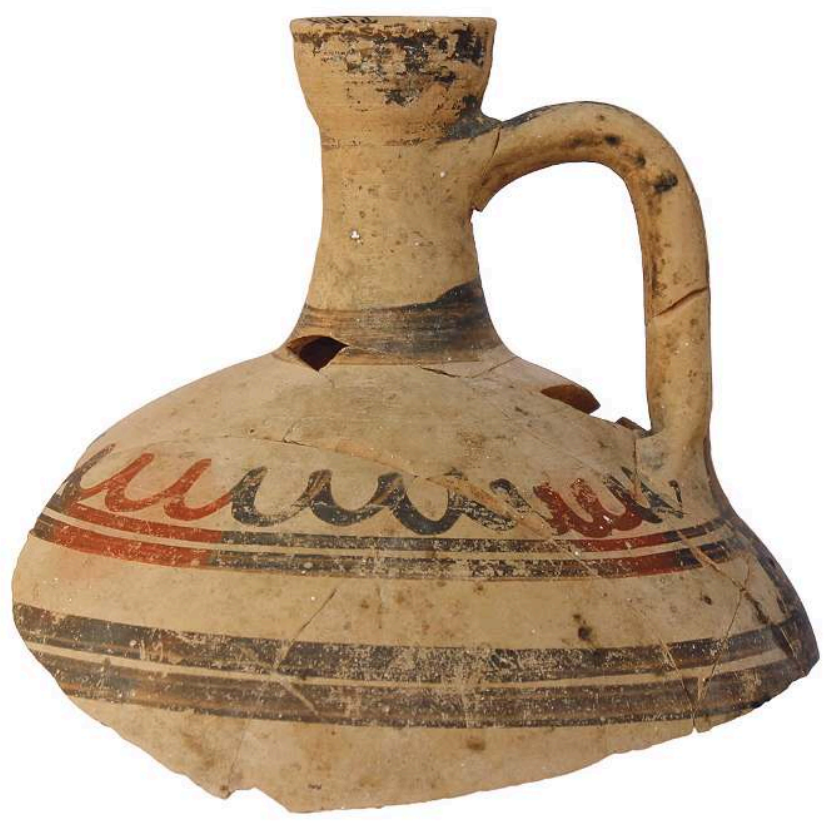

\subsection{Analyses pétrographiques}

Malgré l'absence d'activités sur le terrain, la mission de Tell el-Herr a contribué, cette année, au développement du programme de recherche (AAP 17122) mené conjointement par Sylvie Marchand, Éric Goemaere et $C$. Defernez, sur le thème intitulé «Conteneurs de transport égyptiens du III millénaire av. n.è. à la fin de la période ptolémaïque. Imitations, assimilations et transpositions de modèles étrangers ». À partir de la collecte des échantillons d'argiles calcaires prélevés sur des vases d'origine égyptienne, de grande et de faible capacité - amphores, récipients de service, flacons et bouteilles -, l'identification et la caractérisation des pôles majeurs de consommation et de production de ces emballages, considérés dans leur phase initiale comme des répliques de conteneurs étrangers, égéens ou levantins, ont progressé - notamment grâce aux recherches conduites par É. Goemaere, au sein de l'Institut royal des sciences naturelles de Belgique, à Bruxelles. Le site de Tell el-Herr ayant livré nombre d'attestations de ces vases ${ }^{15}$ est, rappelons-le, l'un des pourvoyeurs essentiels des modèles génériques bien datés des $\mathrm{V}^{\mathrm{e}}$ et $\mathrm{IV}$ e s. av. J.-C. - comme ses pendants cardinaux, Naucratis/Thônis, Memphis/Saqqara, centres économiques majeurs du Delta de la deuxième moitié $\mathrm{du} \mathrm{I}^{\mathrm{er}}$ millénaire. Une collecte de tessons venus des horizons les plus divers de la Méditerranée orientale, principalement alimentée par le site, effectuée au cours des dernières saisons, devrait permettre aux spécialistes une étude affinée et comparée : à défaut d'analyses physico-chimiques non envisageables au Caire, un tel examen ${ }^{16}$ est essentiel, en vue d'une confrontation des résultats acquis avec des données macroscopiques externes déjà connues. 
10 Toujours dans le cadre de ce programme a également été privilégiée l'étude des productions locales, majoritairement attestées dans les niveaux précoces du tell, et non identifiées à ce jour dans d'autres localités $\left(\mathrm{V}^{\mathrm{e}} \mathrm{s}\right.$. - phases VII et VI). L'examen préliminaire du lot de tessons prélevé à l'automne 2019, récemment mené par É. Goemaere, semble bien accréditer le postulat avancé l'an dernier: l'existence de structures d'activités de potiers - et autres structures artisanales - dans l'agglomération de Tell el-Herr ou, à défaut, dans le voisinage de Péluse/Tell Farama, dans tous les cas, dans une zone lacustre/lagunaire, riche en argiles marno-calcaires et dépôts sableux. L'environnement géologique immédiat de ces deux sites, marqué de zones humides - fluviales et lagunaires -, à l'apogée de leur occupation, devait offrir un cadre favorable au prélèvement de telles sources d'argile et à l'implantation de fabriques de poteries. Des études complémentaires programmées à l'automne $2021^{17}$ apporteront sans nul doute davantage d'informations.

\subsection{Vaisselles domestiques et pratiques culinaires}

D'après un rapport d'Aline Banaszak

11 Membre actif de la mission depuis l'automne 2017, Aline Banaszak a entamé une thèse de doctorat à l'automne 2020, à l'université de Lille 3, sous la co-direction de Didier Devauchelle et de C.Defernez. Ce travail de recherche, intitulé «La céramique domestique, témoin des pratiques culinaires en Égypte du premier millénaire av. n.è. », inclut une part importante de la documentation céramique de Tell el-Herr, encore inédite. Les vestiges d'habitats dégagés au cours des dernières années de fouille sur le site y sont nombreux et ont livré un abondant mobilier domestique ${ }^{18}$ (fig. 7). Découvert in situ ou épars sur les sols des pièces (fig. 8), parfois dans de vastes dépotoirs, ce matériel reflète autant les habitudes et traditions égyptiennes que les influences étrangères (achéménides, levantines, grecques et chypriotes). Il comprend une large gamme de céramiques culinaires - pots, jarres, marmites, plats et plaques de cuisson -, et un large panel de céramiques dévolues au stockage et à la préparation alimentaire : les bols-jattes, aussi couramment nommés goldfish bowls, les mortiers et/ou cuvettes, les plaques et moules à pain, les plateaux-dokka, les bassines, les jarres-faisselles, auxquels il faut ajouter les braseros/réchauds, les supports et les couvercles, dominent en effet les assemblages. Réalisées dans une pâte alluviale à dégraissant végétal important et à forte porosité, ces poteries sont habituellement de facture grossière et leur aspect, lourd et massif; leur façonnage s'effectue soit par un modelage manuel, soit à l'aide d'un tour de potier, un tour lent bas aussi communément appelé tournette. 
Fig. 7. Répartition des catégories céramiques du secteur ouest, phase VA (dessin A. Banaszak).

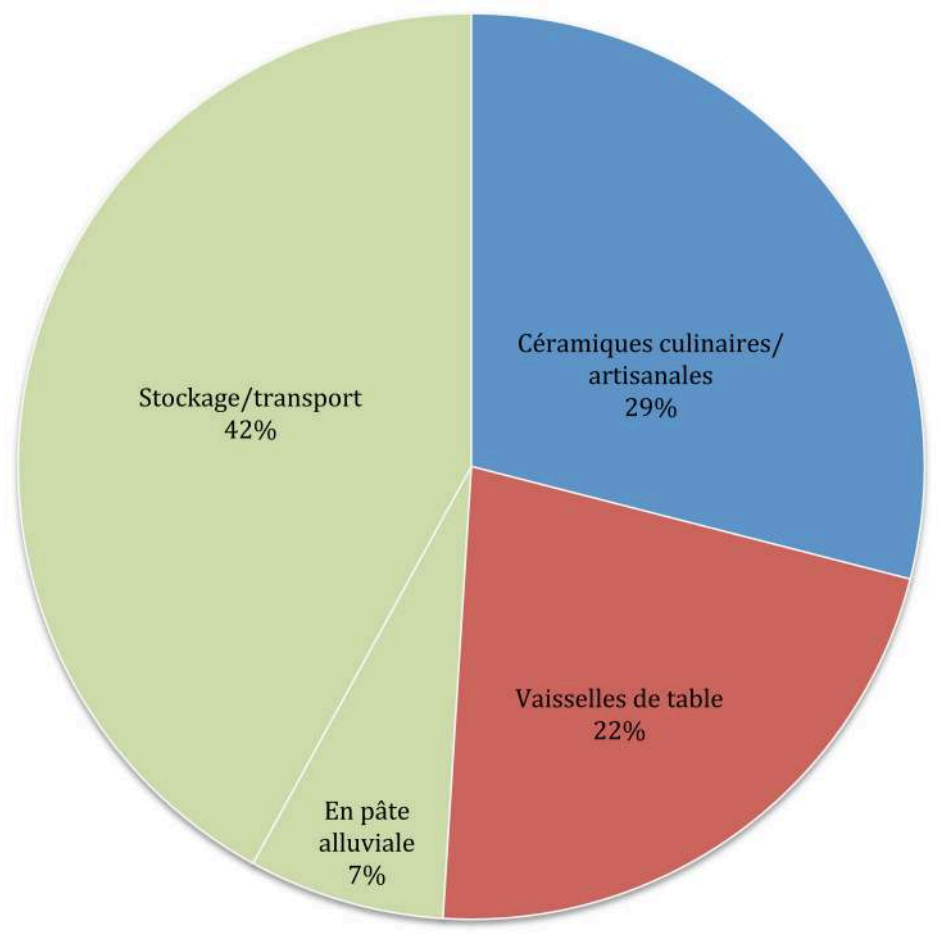

(c) Ifao. 19117_2020_NDMCN_002

Fig. 8. L'espace N139 (îlot IX, unité 31, pièce B) et le matériel associé, phase VA (G. Nogara, A. Banaszak).

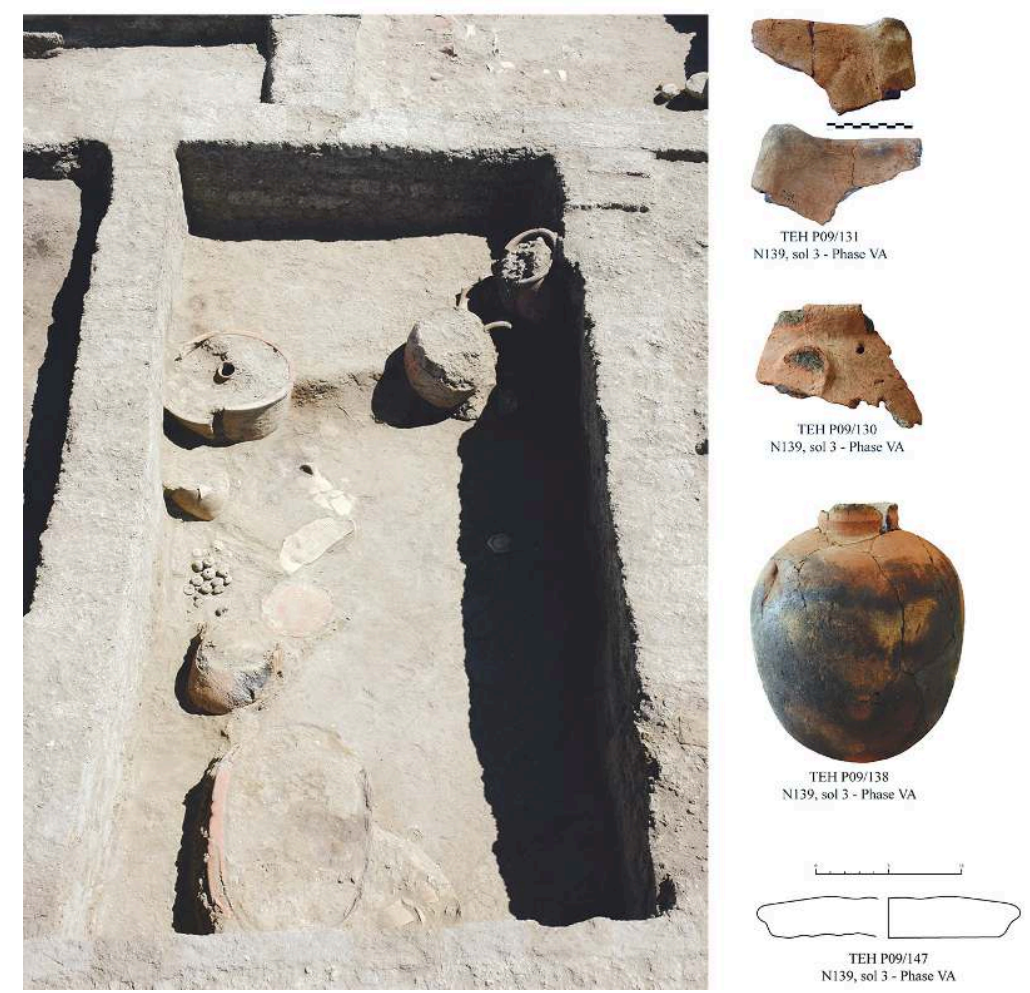

(C) Mission franco-égyptienne de Tell el-Herr/Ifao. 19117_2020_NDMPM_005 

vaisselles de tradition artisanale restent peu documentées: les publications se contentent souvent de les mentionner sans s'attarder sur leurs spécificités. Leur intérêt chrono-typologique peu significatif pour nombre de spécialistes, archéologues et historiens, n'a sans doute guère favorisé leur étude exhaustive, ce qui les a souvent privées d'une description rigoureuse et d'un relevé précis.

13 Pour pallier cette lacune documentaire, l'une des principales finalités de la recherche menée par A. Banaszak est de tenter de définir la fonction réelle, sinon initiale, de plusieurs des catégories de céramiques utilisées par les occupants de la garnison de Tell el-Herr, au sein de leur foyer, par le biais de l'étude de leur contexte archéologique, de leurs caractéristiques techno-morphologiques et de l'analyse comparative avec le matériel extrait d'autres sites contemporains.

Lors des deux dernières campagnes (2018-2019), l'intérêt s'est porté sur des formes particulières, en pâte alluviale, liées essentiellement aux préparations alimentaires (fig. 8) :

- les bassines : larges récipients ovalisés (diamètre souvent supérieur à $60 \mathrm{~cm}$ ) façonnés à la main, à l'aide du colombin ;

- les plaques à pain (ou plateaux), correspondant à des plaques épaisses grossièrement façonnées, de forme ovale ou circulaire ;

- les fours domestiques de petites dimensions, identifiables presque exclusivement grâce à des tessons décorés de larges bandes d'argile digitées ;

- les braseros ou réchauds, dont seuls des bords ont pour l'instant été identifiés ;

- les bassines à marli ou chamber-pots : récipients cylindriques reconnaissables à leur lèvre étalée et leur base annulaire.

Ces sessions d'étude ont permis de mieux appréhender les caractéristiques et l'emploi hypothétique de ces récipients au sein des constructions de Tell el-Herr ${ }^{19}$, et les typologies déjà établies ont pu être complétées ${ }^{20}$. À ce jour, plus d'une centaine de céramiques, issues notamment des îlots d'habitations de la phase VA (fin $\mathrm{v}^{\mathrm{e}}$-premier quart du IV ${ }^{\mathrm{e}}$ s. av. J.-C.), ont pu être recensées et documentées ${ }^{21}$ (fig. 7). Au cours de l'année 2020, des avancées notables ont notamment été faites dans la catégorie réunissant les petits fours d'appoint, dont l'identification restait encore incertaine en raison de leur état fragmentaire élevé ${ }^{22}$. Un examen minutieux de plusieurs fragments joint à l'étude détaillée d'un exemplaire quasi-complet, récemment documenté, a permis de préciser leur morphologie et d'établir une première approche de leur fonction. Les tessons inv. P09/131 (fig. 9) et inv. P09/132 ont révélé de nouveaux éléments quant à l'élévation de ces céramiques : il est apparu, en effet, que la partie sommitale de ces fours devait être largement ouverte et se terminait par une lèvre modelée en biseau. D'autres pièces ont confirmé deux modes différents d'application du décor digité (fig. 10), la présence de plusieurs perforations (évents) dans leur paroi ainsi que des traces de feu sur leur surface interne.

Si l'apport de nouvelles données tend bien à confirmer l'emploi de ces récipients à des fins domestiques et culinaires, certains indices étayés par des essais expérimentaux semblent exclure une corrélation de ces fours avec la cuisson du pain, en particulier avec les plaques à pain trouvées en nombre dans les mêmes contextes ${ }^{23}$ (fig. 8). Les études comparatives révèlent notamment une parenté avec des récipients de tradition hellène. 
17 Ces recherches préliminaires sont présentées dans une contribution du prochain volume des Varia de Tell el-Herr, dédié au mobilier du site et de sa périphérie ${ }^{24}$.

Fig. 9. Bord d'un four d'appoint à décor digité (inv. P09/131, A. Banaszak).

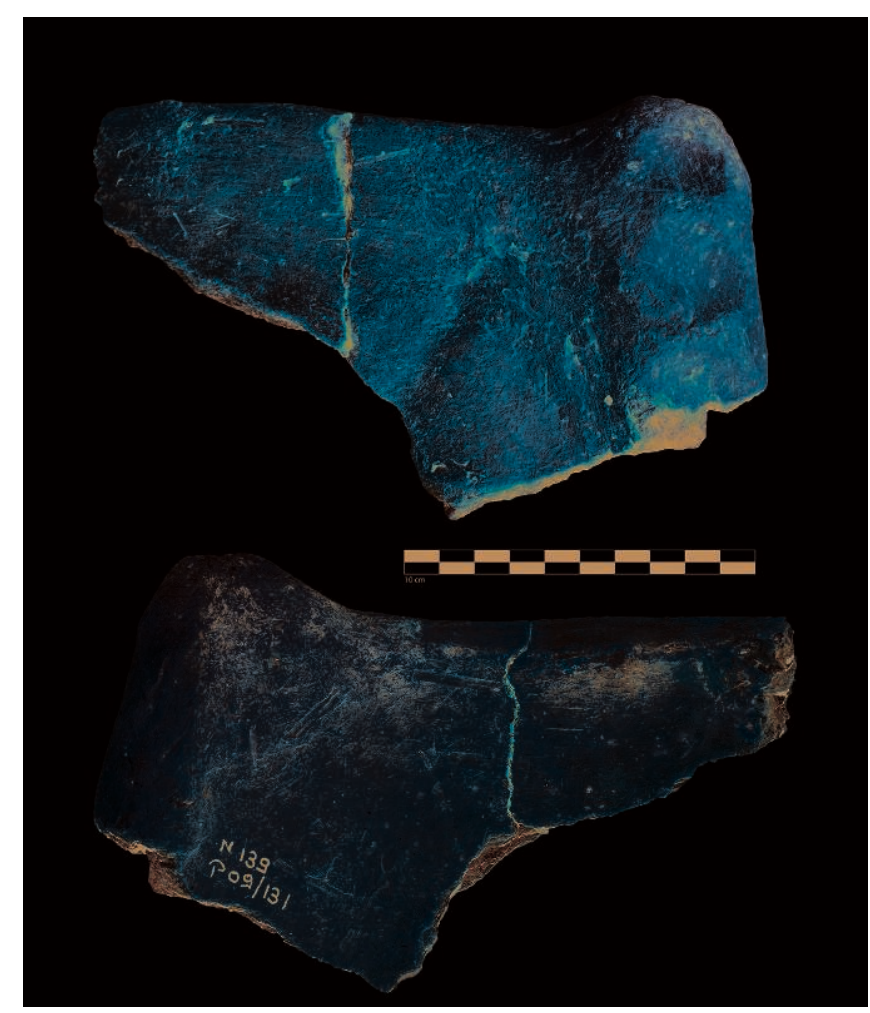

(C) Ifao. 19117_2020_NDMPM_006 
Fig. 10. Éléments de four à décor digité (inv. P00/423, A. Banaszak).

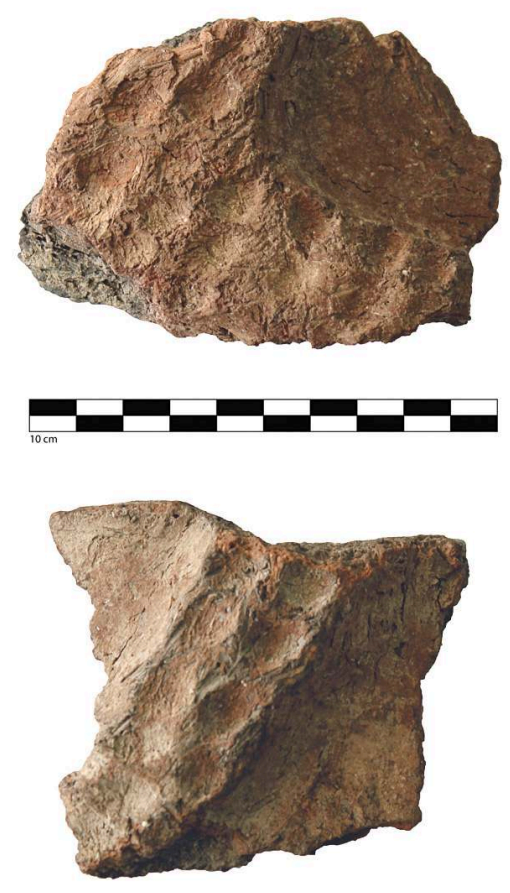

(C) Ifao. 19117_2020_NDMPM_007

\subsection{La céramique domestique : perspectives de recherche}

Malgré le travail important accompli lors des dernières missions, un matériel domestique encore abondant et digne d'intérêt reste à étudier. Plusieurs catégories de céramiques n'ont pas encore été documentées ou n'ont pas encore pu bénéficier d'une étude approfondie. La fonction de la plupart des vaisselles demeure hypothétique et des typologies formelles de certains groupes doivent être établies, afin de mieux comprendre leur présence au sein des habitations des différentes phases d'occupation du site.

De manière générale, une vision plus globale de la «batterie de cuisine » traditionnelle de Tell el-Herr doit être définie pour entamer une étude comparative précise avec la documentation analogue issue d'autres sites du Delta et d'autres régions de l'Égypte, du Levant et de l'Égée. Ce travail devrait permettre, à terme, l'insertion des ensembles culinaires connus dans une réalité archéologique et territoriale.

À cet égard, un recours à l'archéologie expérimentale s'impose, afin de valider ou invalider les fonctions hypothétiques des vases identifiés. Ce projet porte en priorité sur les catégories formelles déjà bien circonscrites: par exemple, dans le cadre de la reconstitution des fours d'appoint domestiques et des éléments vaisseliers qui lui sont habituellement associés, il s'agirait de recréer les gestes de façonnage de l'artisan et de confronter les hypothèses de travail à la mise en pratique. L'expérimentation gagnerait cependant à être étendue à d'autres formes, les plateaux à pain notamment et bien d'autres vaisselles atypiques. Il est également prévu de couler des moulages de plâtre ou de silicone sur les plaques présentant des motifs incisés distinctifs, afin de vérifier 
l'emprise du décor sur la galette de pain ${ }^{25}$. Dans une phase ultérieure, il serait intéressant d'en façonner plusieurs exemplaires et de procéder à des tests de cuisson ${ }^{26}$. Quelques tentatives ont déjà été menées afin de mieux définir les besoins et conditions des expérimentations à venir.

\section{2. Étude du petit mobilier archéologique : amulettes, perles et bijoux}

(d'après les rapports de Sépideh Qaheri et Nathalie Favry)

Découverts à foison dans tous les niveaux du site, les amulettes, perles et bijoux dominent largement le petit mobilier archéologique : 743 objets ont été recensés sur un nombre total de 893, au cours des précédentes missions. Cependant, dans l'optique de la préparation du prochain volume de synthèse sur les niveaux récents du tell (phases VBVA, dernier quart du $v^{e} s$. et $\mathrm{IV}^{\mathrm{e}} \mathrm{s}$. av. J.-C.), seuls les artefacts issus des contextes assurément datés de ces périodes (384 objets) ont bénéficié, au cours de l'année, d'une étude affinée (fig. 11) : description typologique, quantification, étude comparée, outre la vectorisation des dessins.

Fig. 11. Répartition des amulettes oudjat de Tell el-Herr, depuis 1986 (S. Qaheri).

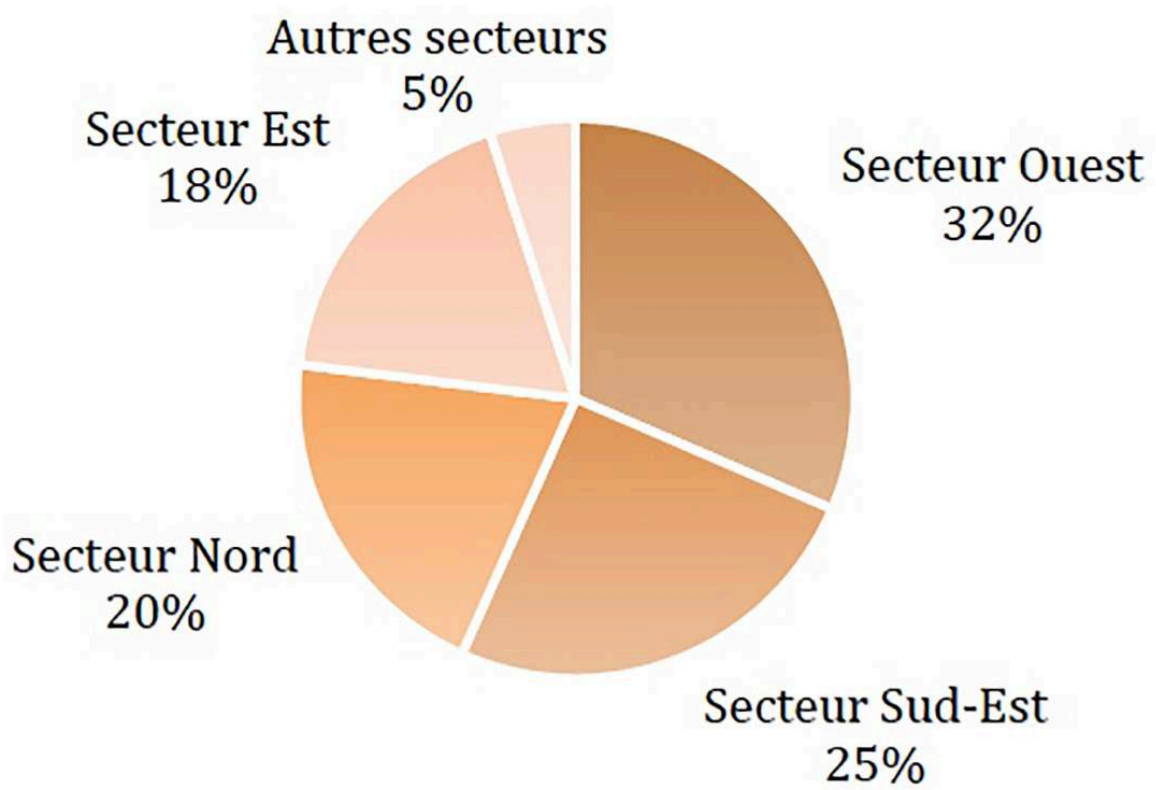

(C) Ifao. 19117_2020_NDMCN_003

De par leur grand nombre, les amulettes ont suscité une attention particulière (fig. 12-14). Et comme dans d'autres sites contemporains, les plus répandues sont les amulettes de Bès et les amulettes d'œil-oudjat, dont la taille, la forme générale et le traitement offrent une grande diversité ; selon leur module et leur aspect (perforation simple ou double), elles se répartissent entre simples talismans (apotropaïques) et écarteurs de colliers. Leur variété iconographique est également manifeste et dénote 
leur importance dans le quotidien des occupants du site, tout groupe ethnique confondu. À côté des modèles sommaires sont attestées des pièces plus sophistiquées, de belle facture, qui témoignent d'une finesse et d'une précision dans leurs traitement et exécution (fig. 12). Des versions un peu éloignées des types classiques nécessitant une analyse fine paraissent bien confirmer l'originalité du matériel de Tell el-Herr et, paradoxalement, l'uniformité de certaines productions sous l'Empire perse achéménide: une étude spécifique sur une amulette Bès (inv.03/087) met en avant cette uniformité27. Quelques pièces laissent augurer, en effet, des interactions culturelles marquées entre les différents contingents en place dans la région.

Sous l'expertise de Sépideh Qaheri, l'étude de ces deux talismans protecteurs, bien documentés à l'époque perse en Égypte, au Levant et au Proche-Orient, s'est poursuivie en 2020. L'inventaire précis de ces amulettes, à partir des données consignées dans la base documentaire de la mission, a progressé, en vue d'un examen complet des pièces listées au cours de la prochaine campagne. Le répertoire typologique pour chaque groupe défini a pu être complété, de même que les fichiers photographiques associés, établis à l'aide du logiciel Adobe Bridge ; selon le schéma IPTC Core, plus de 400 fichiers photos et dessins réunissent, à ce jour, les métadonnées de chaque objet identifié (informations de documents numériques, données de fouilles, occurrences, références chronologiques, etc.) (fig. 12). Au terme de ce travail, plusieurs grands types et soustypes pour chacun des ensembles identifiés sont apparus.

L'inventaire des pièces sera complété lors des prochaines missions, dans les magasins des antiquités du MoTA de San el-Haggar, une fois leur analyse et leur couverture photographique haute-résolution achevées. Leur étude intégrale devrait aboutir à l'établissement d'un catalogue chrono-typologique de référence prenant en compte les données matérielles fournies par d'autres sites en Égypte et au-delà ; ce qui ouvrira vraisemblablement d'autres perspectives de recherche. Les travaux menés antérieurement ont récemment abouti à la préparation d'une étude dans le prochain volume collectif de la mission ${ }^{28}$. Les résultats des investigations futures devraient alimenter bien d'autres contributions mettant en exergue l'apport de ce petit mobilier à l'archéologie et à l'histoire de l'Égypte achéménide. 
Fig. 12. Traitement informatique des fichiers photos de Tell el-Herr (missions 1986-2018), avec les exemples d'amulettes oudjat du type A.1.1 unicolore (S. Qaheri).

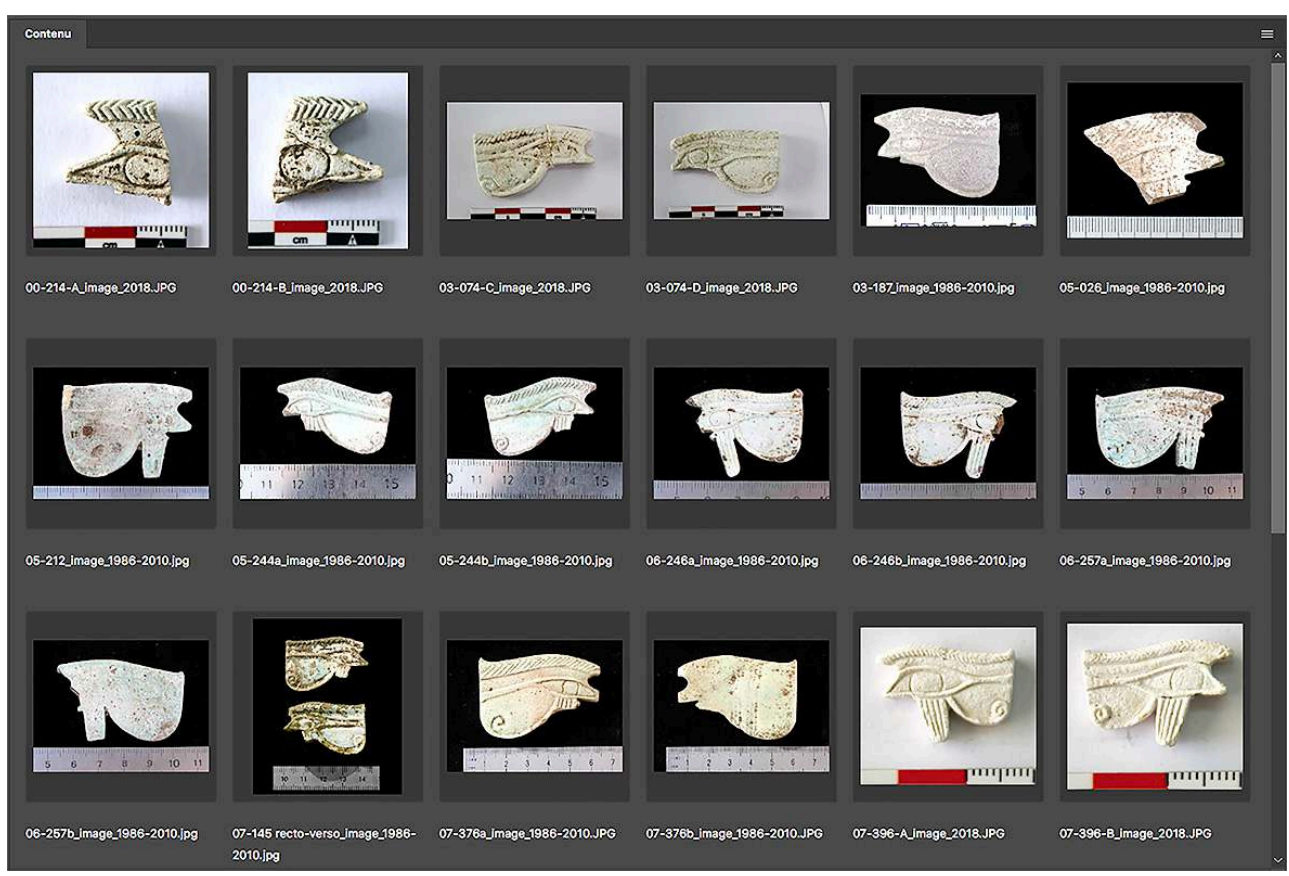

(C) Ifao. 19117_2020_NDMPM_008

Fig. 13. Amulette œil oudjat en pâte calcaire glaçurée bleue - secteur nord, fin phase VA (inv. 01/262, R.-P. Dissaux).

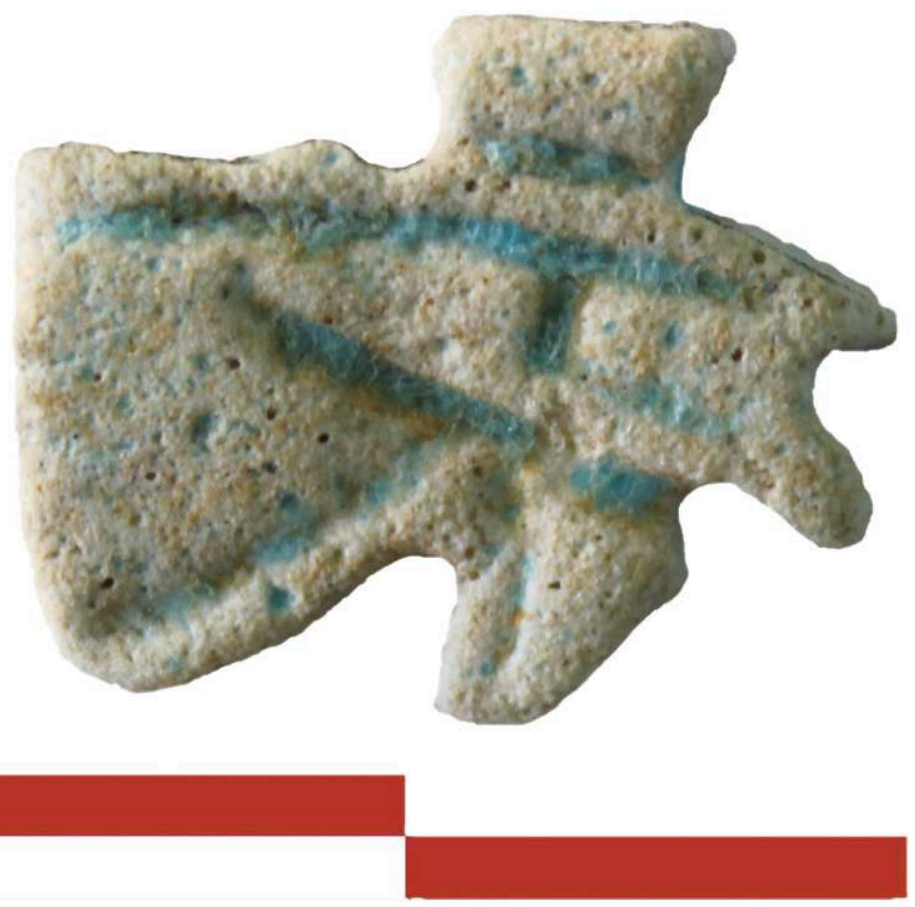

(C) Ifao. 19117_2020_NDMPM_009 
Fig. 14. Moule en calcaire d'une divinité (?) assise - secteur est, E29 (inv. 09/026, J.-M. Yoyotte).

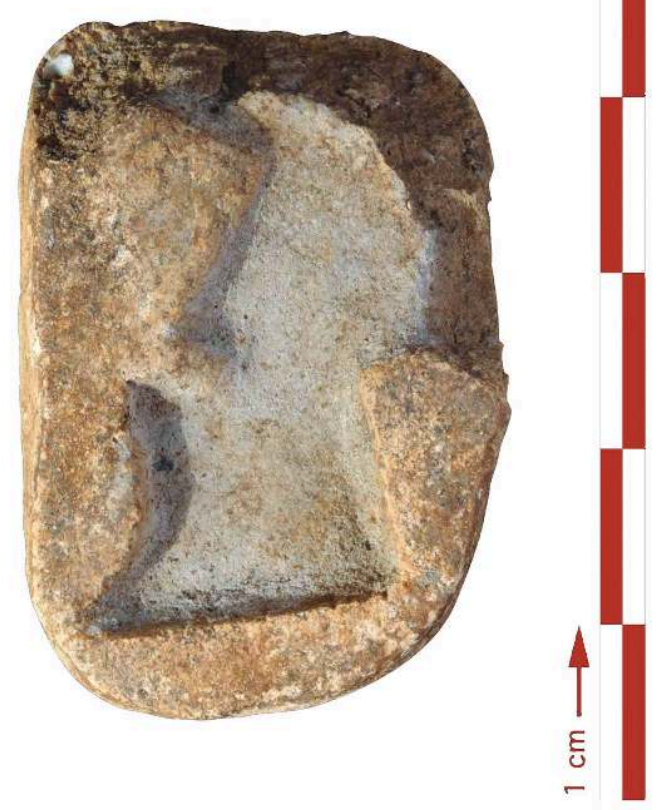

\section{(C) Ifao. 19117_2020_NDMPM_010} apport documentaire. L'examen exhaustif des perles et pendentifs (et autres éléments de parure), entamé l'an dernier, s'est poursuivi. À l'exemple des amulettes, une diversité de matière (cornaline, calcaire, pâte de verre, os, pierre), de taille et de forme (plate, sphérique, tubulaire, discoïde), caractérise la collection attribuée aux phases récentes du tell (environ 150 perles pour les phases concernées, VB et VA) - comme dans bien d'autres localités. Cependant, comme il a été indiqué29, l'originalité de ce mobilier réside dans la présence de belles trouvailles phéniciennes dont les plus significatives, constituées de perles à ocelles (colliers) et de masques-pendentifs à figures, ont nécessité une étude comparée plus approfondie; d'autant que plusieurs exemples proviennent de contextes assurés et bien datés des phases d'occupation du tell en cours de publication. On sait, par le biais d'une documentation pléthorique, que les Phéniciens ont excellé dans cet art, et les diverses études les concernant en offrent de multiples témoignages : les sites de Sidon, de Beyrouth, de Byblos et de Carthage livrent une documentation comparable, richement illustrée et commentée. En plus de confirmer l'identité culturelle de ces pièces, ces travaux apportent de plus amples précisions sur leur mise en œuvre (aspects techniques de leur production) - selon la technique "sur noyau» de sable--, sur leur conception, leur répartition et leur évolution chronologique.

Parallèlement à cette recherche, une nouvelle analyse des quelques trouvailles issues des niveaux ptolémaïques et romains dégagés à la suite des deux publications majeures qui leur a été consacrées, mais non cataloguées dans ces volumes, a été menée. Une contribution commune avec Séverine Marchi, dans le prochain volume collectif de la mission $^{30}$, présentera les résultats acquis à l'issue de l'examen de ce matériel, constitué 
de plus d'une quarantaine d'objets, dont une trentaine provenant de dix-sept niveaux d'occupation romains (fig. 15-17) ; une quinzaine de pièces extraites de contextes moins assurés a également été étudiée.

27 Comme dans d'autres sites de la région, la catégorie des bijoux, constituée d'une large collection d'anneaux/boucles d'oreille, de bracelets (fig. 16), de fibules (fig. 17), de bagues et/ou chatons de bague (fig. 18), d'éléments de collier ou autres éléments de parure, en métal et en pierre semi-précieuse, est importante à Tell el-Herr et suscite un intérêt notable de par la rareté et la variété de ses composants.

Fig. 15. Scarabée en pierre noire (droite ?) - camp romain (inv. 88/220, N. Favry).
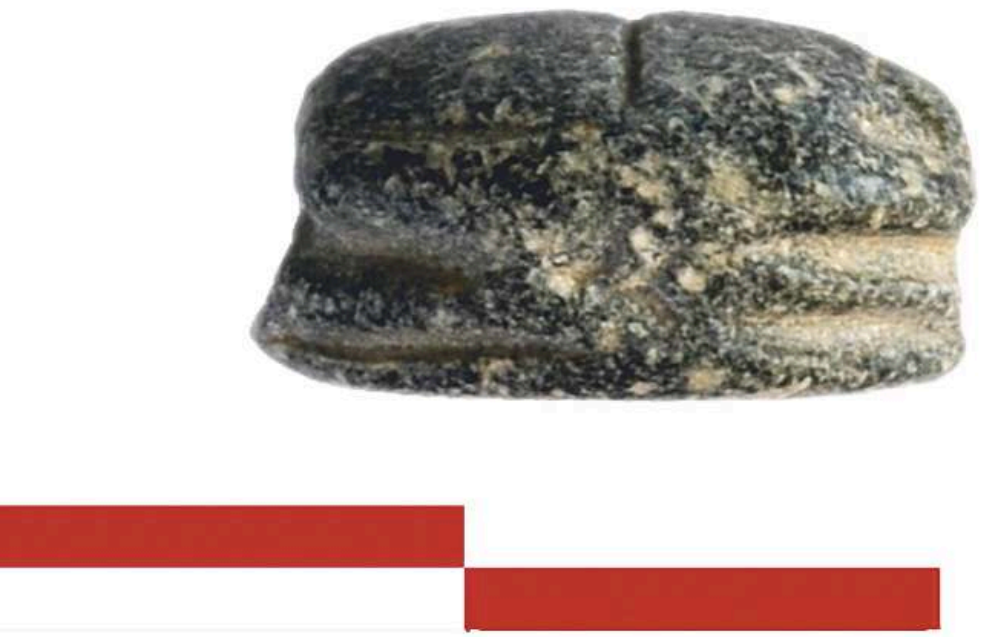

(c) Ifao. 19117_2020_NDMPM_011 
Fig. 16. Bracelet fragmentaire en os - sondage nord, C4, Bas-Empire (inv. 94/055, N. Favry).

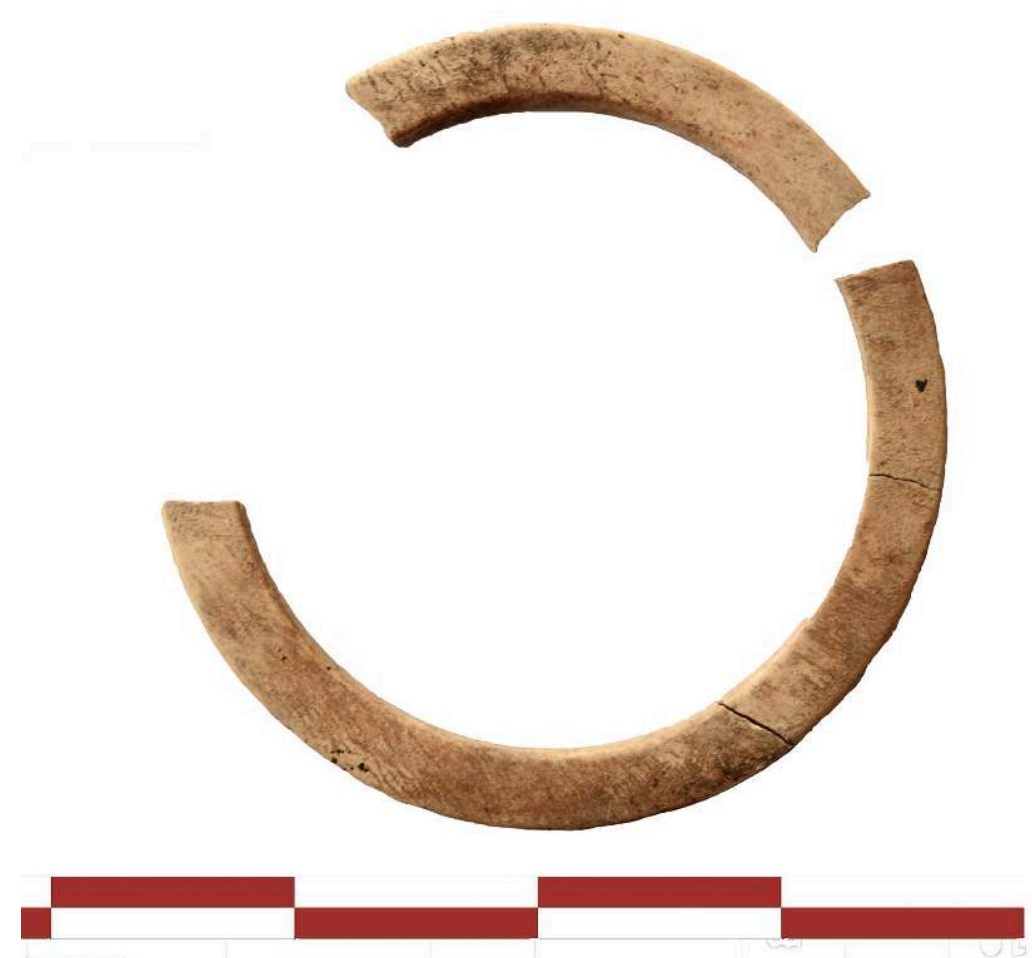

(C) Ifao. 19117_2020_NDMPM_012

Fig. 17. Fibule fragmentaire en bronze - secteur nord, N9, phase VA (inv. 98/120, N. Favry).

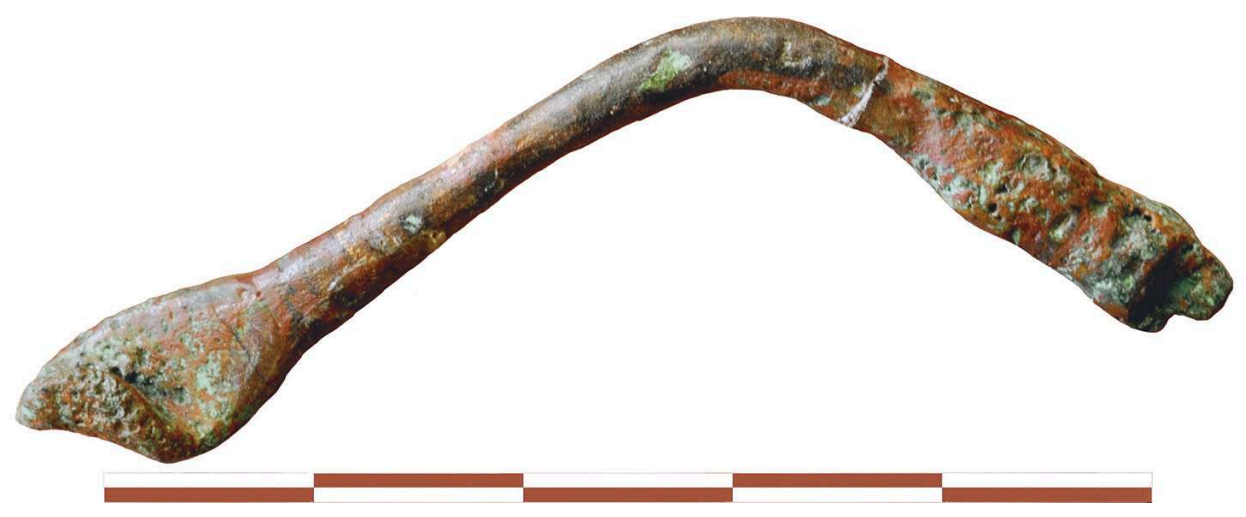

(C) Ifao. 19117_2020_NDMPM_013 
Fig. 18. Chaton de bague en bronze inscrit - secteur est, phase IV (inv. 88/181, N. Favry).

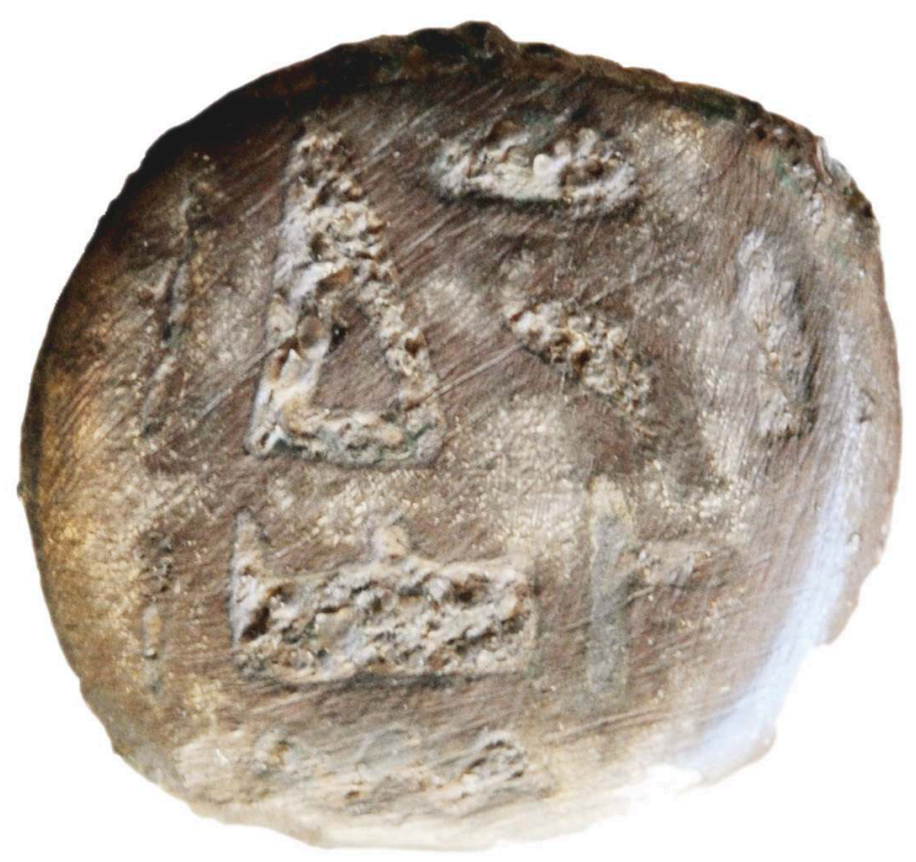

\section{Autres études}

\subsection{La coroplathie}

Sous la responsabilité de Pascale Ballet, en collaboration avec Élodie Rotté ${ }^{31}$, les recherches ont progressé sur la thématique de la petite plastique de calcaire et de terre cuite. Le corpus perse et $d u \mathrm{IV}^{\mathrm{e}}$ s. av. J.-C. s'est enrichi de nouvelles données, dans les catégories préalablement évoquées ${ }^{32}$ : au catalogue initialement établi se sont ajoutées de nouvelles images de la piété individuelle et/ou collective, de cavaliers et/ou équidés, de phallus, de Bès et de Silènes, etc. Plusieurs d'entre elles connaissent des contreparties dans les grands centres de consommation et de production du Delta et de régions voisines, d'autres possèdent des occurrences dans les corpus connus du monde méditerranéen oriental, qui confirment sans conteste leur datation commune. Si les images de la fécondité, femmes nues sur " plaquette » ou dans un naos, dont des pièces exceptionnelles $^{33}$ et bien préservées ${ }^{34}$ sont à signaler (fig. 19), émergent largement des assemblages connus, les cavaliers "scythes ", barbus et coiffés du bonnet conique, plus connus sous l'appellation de "cavaliers perses» (fig. 20), sont incontestablement les témoignages les plus éloquents d'une certaine uniformité de cet artisanat d'un point à l'autre du territoire achéménide. Leur large diffusion dans l'Empire est désormais notoire. 
D'une manière générale, la petite plastique de Tell el-Herr traduit une société pluriethnique « oscillant entre égyptianité et ouverture sur la Méditerranée ». Et sur ce point, Tell el-Herr demeure un site d'une importance capitale pour aborder les différents aspects de la petite statuaire de la fin de la Basse Époque - à l'instar des autres pôles récepteurs et consommateurs, majeurs, de la période concernée (Naucratis, Thônis/Héracléion, Bouto, Mendès, Tanis et Memphis).

Il est à préciser, cependant, que si quelques terres cuites figurées paraissent bien correspondre à d'authentiques importations du monde grec - sinon égéen -, du Levant ou de Chypre, la plupart réunissent les critères d'une production locale et/ou régionale, dont l'origine précise reste à déterminer: les propriétés techniques de leur fabrique alliées au caractère fruste de leur mise en œuvre - pièces moulées ou modelées, selon les groupes définis - plaident en ce sens et semblent en effet favoriser une provenance dans le voisinage immédiat de leur contexte de découverte.

Des compléments d'informations bibliographiques ont également été apportés au riche répertoire iconographique des époques ptolémaïque et romaine déjà bien établi ${ }^{35}$.

Fig. 19. Image de la fécondité, femme nue sur plaque de terre cuite (inv. 84/002, J.-F. Gout).

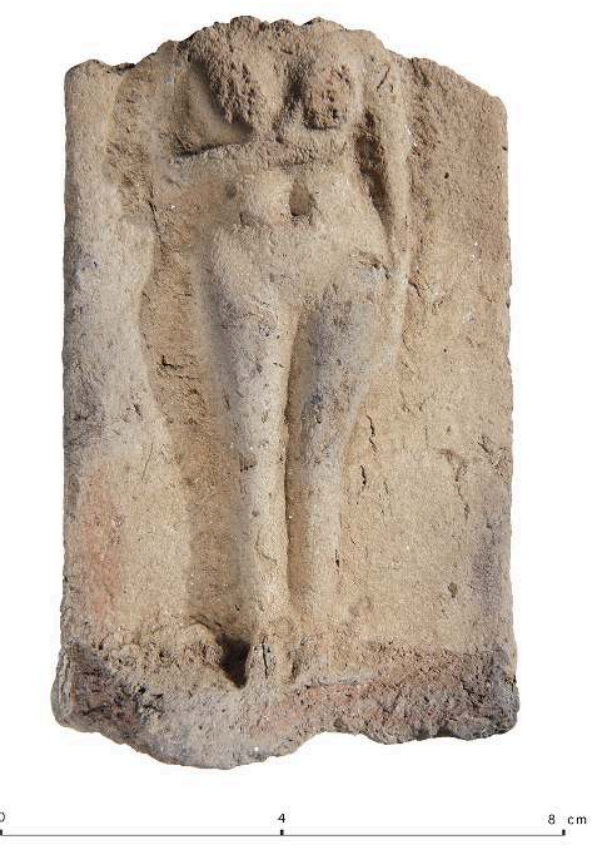


Fig. 20. Tête d'un cavalier perse, pâte alluviale (inv. 87/258, J.-F. Gout).

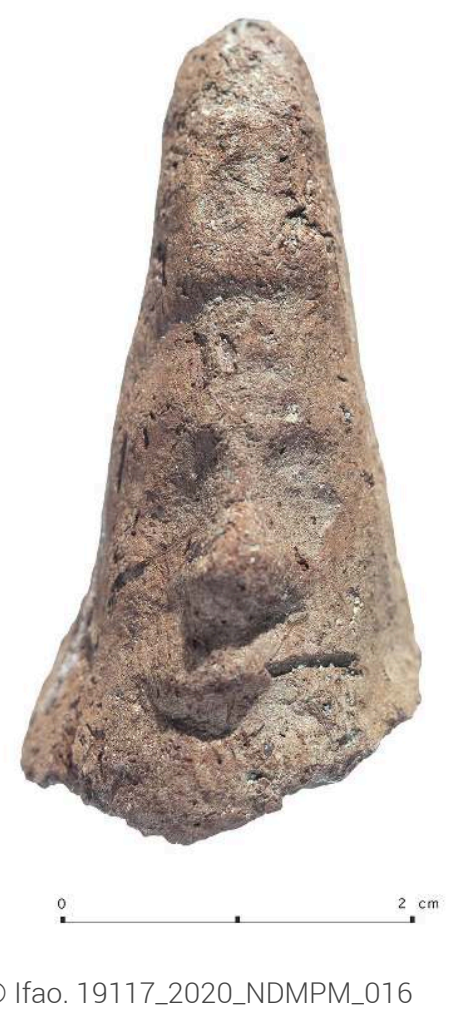

\subsection{Le mobilier lithique}

Comme il a été indiqué dans le précédent rapport ${ }^{36}$, seul l'inventaire du mobilier lithique a pu être achevé lors de la dernière campagne. Son étude a pu être complétée, cette année, par S. Marchi. Ce dossier, assez conséquent (plus d'une centaine de pièces en basalte, calcaire, schiste, diorite et calcite), réunit des données essentielles dont l'apport peut être capital pour une meilleure compréhension du cadre de vie quotidien des occupants du site. À travers les éléments significatifs de ce matériel sont mises en avant diverses activités pratiquées dans la garnison : construction et bâti (éléments de portes, crapaudines, etc.), activités artisanales et domestiques (pesons, percuteurs, mortiers, cuves), activités économiques/échanges (poids de balance), activités ludiques (dés, jetons, damiers), sans oublier les cérémonies festives, cultuelles et/ou religieuses ; les vaisselles d'apparat - plats de service, vases à parfum ou à onguents/alabastres -, ainsi que les encensoirs sont bien représentés. Un catalogue exhaustif de l'ensemble des pièces retenues, dont plusieurs sont amplement référencées et documentées au Levant, en Syrie, en Grèce et à Chypre, est en cours d'élaboration.

\section{Formation et coopération}

C. Defernez

De 2016 à 2020, depuis la reprise des activités de la mission dans le Delta, la coopération scientifique entre les membres de la mission et les responsables des inspectorats du Nord-Sinaï et de Qantara s'est maintenue. Plusieurs d'entre eux ont contribué à la 
valorisation de leurs recherches en les encadrant dans leurs travaux de Master et de thèse. Soutenue en 2019, à la faculté des lettres de Sorbonne Université, la thèse de doctorat de Elsayed Abd el-Alim, intitulée Tell Heboua I et II. Enquête archéologique sur deux sites de la frontière orientale de l'Égypte à l'époque saite a été déposée dans la collection Orient \& Méditerranée de l'UMR 8167 pour une parution prochaine en 2021.

Parallèlement à ce travail universitaire, Elsayed Abd el-Alim, actuellement directeur de l'inspectorat du MoTA de Qantara-Est, poursuit la valorisation de ses recherches sur les niveaux tardifs de Tell Heboua I, II et III, dans le cadre d'articles et de communications au sein de congrès et tables-rondes internationales (voir infra). En maintenant ses opérations de terrain sur les sites majeurs du nord du Sinaï, il demeure un membre actif de la mission de Tell el-Herr et, surtout, un acteur essentiel dans la coopération entre les inspectorats du Nord-Sinaï, de San el-Haggar et de Zagazig.

\section{Publications de l'équipe (2019-2021)}

- Elsayed Abd el-Alim, «Tell Habua: New Discoveries from the Saite Period », EgArch 56, 2020, p. 10-15.

- Elsayed Abd el-Alim, Tell Heboua I et II. Enquête archéologique sur deux sites de la frontière orientale de l'Égypte à l'époque saïte, Paris, à paraître.

- Mohamed Abd el-Maksoud, ElSayed Abd el-Alim, «Les magasins royaux récemment découverts à Tell Heboua II-le khétem de Tjarou ", in Catherine Defernez, Nathalie Favry (éd.), Tell el-Herr. Varia I, NeHet 7, à paraître.

- Pascale Ballet, Figurines et société de l'Égypte ptolémaïque et romaine, Collection Antiqva 17, Paris, Picard, 2020.

- Pascale Ballet, «Figurines, sites et contextes dans l'Égypte gréco-romaine. Études de cas provinciaux : de Tell el-Herr (Sinaï) à Bouto ", dans un volume de Mélanges, Varsovie, sous presse.

- Aline Banaszak, «La céramique domestique de Tell el-Herr. Présentation d'une forme particulière : le four cylindrique à décor digité », in Catherine Defernez, Nathalie Favry (éd.), Tell el-Herr. Varia I, NeHet 7, à paraître.

-Jean-Yves Carrez-Maratray, «Retour sur l'Antinoos de Péluse », in Catherine Defernez, Nathalie Favry (éd.), Tell el-Herr. Varia I, NeHet 7, à paraître.

- Louis Chaix, "Un "basset" byzantin à Tell el-Farama (Sinaï, Égypte)», in Sandrine Vuillemier, Pierre Meyrat (éd.), Sur les pistes du désert. Mélanges offerts à Michel Valloggia, Gollion, Infolio éditions, 2019, p. 27-34.

- Louis Chaix, «Le monde animal à Tell el-Herr », in Catherine Defernez, Nathalie Favry (éd.), Tell el-Herr. Varia I, NeHet 7, à paraître.

- Catherine Defernez, «Quelques productions phéniciennes marginales découvertes à Tell elHerr », BCE 29, 2019, p. 5-68.

- Catherine Defernez, «Compte rendu de S. Lupo, Tell el-Ghaba III. A Third Intermediate-Early Saite Period Site in the Egyptian Eastern Delta, BAR-IS 2756, Oxford, 2015 », AntOr 17, 2019, p. 276-287.

- Catherine Defernez, «L'image de Bès et la céramique égyptienne », EAO 100, 2020-2021, p. 47-58.

- Catherine Defernez, "Tell el-Herr », in Laurent Coulon, Mélanie Cressent (éd.), French Archaeology in Egypt: Research, Cooperation, Innovation, BiGen 62, Le Caire, Ifao, 2020, p. 78-81 (version arabe, BiGen 61). 
- Catherine Defernez, «D'Amathonte au Nord-Sinaï : sur les traces d'un scarabée tétraptère ", in Antigone Marangou, Thierry Petit (éd.), Le Palais d'Amathonte des origines à la fin du royaume. État des recherches, Archéologie \& Culture, Rennes, Presses universitaires de Rennes, sous presse.

- Catherine Defernez, «Le maintien de la tradition saïte dans l'industrie céramique de la fin de l'époque perse achéménide ", in Catherine Defernez, Nathalie Favry (éd.), Tell el-Herr. Varia I, NeHet 7, à paraître.

- Catherine Defernez, Nathalie Favry, «Tell el-Herr», in Laurent Coulon, Mélanie Cressent (éd.), Archéologie française en Égypte. Recherche, coopération, innovation, BiGen 59, Le Caire, Ifao, 2019, p. 114-119.

- Catherine Defernez, Nathalie Favry (avec les contributions de Pascale Ballet, Aline Banaszak, Anne-Laure Daubisse, Séverine Marchi, Sépideh Qaheri), «Tell el-Herr », Bulletin archéologique des Écoles françaises à l'étranger, 2020, article en ligne sur OpenEdition Journals, https://journals.openedition.org/baefe/772, consulté le 2 mai 2021.

- Delphine Dixneuf, « De Péluse à al-Farama : les objets de la vie quotidienne entre le $\mathrm{IV}^{\mathrm{e}}$ et le $\mathrm{XI}^{\mathrm{e}}$ siècle ", in Catherine Defernez, Nathalie Favry (éd.), Tell el-Herr. Varia I, NeHet 7, à paraître.

- Nathalie Favry, Séverine Marchi, «Les niveaux ptolémaïques et romains de Tell el-Herr revisités ", in Catherine Defernez, Nathalie Favry (éd.), Tell el-Herr. Varia I, NeHet 7, à paraître.

- Sépideh Qaheri, « Note sur une amulette Bès d'époque perse à Tell el-Herr ", in Catherine Defernez, Nathalie Favry (éd.), Tell el-Herr. Varia I, NeHet 7, à paraître.

- Dominique Valbelle, "One More Time, "the Way(s)-of-Horus" ", in Janice Kamrin, Miroslav Bárta, Salima Ikram, Mark Lehner, Mohamed Megahed (éd.), Guardian of Ancient Egypt: Studies in Honor of Zahi Hawass, vol. 3, Prague, Charles University, 2021, p. 1607-1613.

- Dominique Valbelle, "Une stèle de Sobek-Rê "qui écoute les prières" à Tell el-Herr ", in Catherine Defernez, Nathalie Favry (éd.), Tell el-Herr. Varia I, NeHet 7, à paraître.

\section{Valorisation}

Aline Banaszak, « Témoignages archéologiques de la préparation du pain à Tell el-Herr $\left(v^{\mathrm{e}} \mathrm{s} . \mathrm{av} . \mathrm{J} . \mathrm{-C}.\right)$ », poster présenté dans le cadre du colloque intitulé "Premières bouchées de pain: Archéologie et Histoire du "pain" : Europe-Égypte (ProtohistoireAntiquité) », 21-25 octobre 2019, université Paul-Valéry Montpellier 3, organisé par Philippe Marinval, Florian Jedrusiak, Jean-Marc Luce, Adeline Bats).

\section{Communications, colloques nationaux et internationaux}

- Elsayed Abd el-Alim, automne 2019 (Le Caire, Institut culturel italien) : « The Eastern Gate of Egypt during Saite period ».

- Elsayed Abd el-Alim, novembre 2019 (Le Caire, Marriott Mena House), XII ${ }^{\mathrm{e}}$ congrès international des égyptologues : « Casemate Foundation Platform at Tell Heboua I ».

- Aline Banaszak, 5 février 2021 (université de Lille 3), journée d'étude «Réemploi, réutilisation et référence dans les sociétés anciennes»: "Autour de l'attribution fonctionnelle : réutilisation ou multifonctionnalité ? L'exemple de la céramique de Tell elHerr (Nord-Sinaï, $\mathrm{v}^{\mathrm{e}}-\mathrm{IV}^{\mathrm{e}}$ siècles av. J.-C.) ». 
- Catherine Defernez, Dominique Valbelle, (Paris, Académie des inscriptions et belles-Lettres) : «Tell el-Herr (Migdol), un site majeur de l'empire perse en Égypte» (communication programmée le 5 mars 2021).

\section{BIBLIOGRAPHIE}

BALLET 2020

Pascale Ballet, Figurines et société de l'Égypte ptolémaïque et romaine, Antiqua 7, Paris, Picard, 2020.

BANASZAK à paraître

Aline Banaszak, « La céramique domestique de Tell el-Herr. Présentation d'une forme particulière : le four cylindrique à décor digité », in Catherine Defernez, Nathalie Favry (éd.), Tell el-Herr. Varia I, NeHet 7, à paraître.

DEFERNEZ 2001

Catherine Defernez, La céramique d'époque perse à Tell el-Herr. Étude chrono-typologique et comparative, CRIPEL-Suppl. 5/1-2, Lille, université Charles-de-Gaulle - Lille 3, 2001.

DEFERNEZ, NOGARA, VALBELLE 2017

Catherine Defernez, Giorgio Nogara, Dominique Valbelle, Tell el-Herr. Les niveaux de la fin $d u v^{e}$ et $d u$ iv siècle av. J.-C., t. I : Un palais oriental à Migdol, Paris, PUPS, 2017.

DEFERNEZ et al. 2020

Catherine Defernez, Nathalie Favry, avec les contributions de Pascale Ballet, Aline Banaszak, Anne-Laure Daubisse, Séverine Marchi, Sépideh Qaheri, « Tell el-Herr », Bulletin archéologique des Écoles françaises à l'étranger , 2020, article en ligne sur OpenEdition Journals https:// journals.openedition.org/baefe/772, consulté le 1 mai 2021.

FAVRY, MARCHI à paraître

Nathalie Favry, Séverine Marchi, « Les niveaux ptolémaïques et romains de Tell el-Herr revisités ", in Catherine Defernez, Nathalie Favry (éd.), Tell el-Herr. Varia I, NeHet 7, à paraître.

GRATIEN, SOULIÉ 1988

Brigitte Gratien, Daniel Soulié, « La céramique de Tell el-Herr. Campagnes 1986 et 1988. Étude préliminaire », CRIPLEL 10, 1988, p. 23-56.

LEHMANN et al. 2020

Gunnar Lehmann, Yiftah Shalev, Hans Mommsen, David Ben-Shlomo, Malgorzata Daskiewiecz, Gerwulf Schneider, Ayelet Gilboa, «The Kelenderis Pottery workshop(s): Newly Identified Agents in East Mediterranean Maritime Exchange Networks in the Achaemenid Period », Levant 51/3, 2020, p. 1-27.

LOUIS, GRATIEN 1990

Étienne Louis, Brigitte Gratien, « Tell el-Herr. Premières observations sur l'agglomération antique ", CRIPEL 12, 1990, p. 71-84.

MARCHAND 2017

Sylvie Marchand, « Remarques sur les moules à pains et les plaques de cuisson dans l'Égypte ancienne », BCE 27, 2017, p. 223-250. 
MARCHI 2014

Séverine Marchi, L'habitat dans les forteresses de Migdol (Tell el-Herr) durant les $v^{e}$ et iv ${ }^{e}$ siècles avant J.C. Étude archéologique, Paris, PUPS, 2014.

QAHERI à paraître

Sépideh Qaheri, « Note sur une amulette Bès d'époque perse à Tell el-Herr », in Catherine Defernez, Nathalie Favry (éd.), Tell el-Herr. Varia I, NeHet 7, à paraître.

ROTTÉ 2012

Élodie Rotté, « Egyptian Plaques Terracottas of Standing Nude Women from the Late Period:

Egyptian Heritage or Foreign Inluences », Newsletter of the Coroplastic Studies Interest Group 7, 2012, article en ligne, http://www.academia.edu/2233475/

Egyptian_Plaques_Terracottas_of_Standing_Nude_Women_from_the_Late_Period_Egyptian_Heritage_or_Foreign_Influences ${ }_{2}$ consulté le 2 mai 2021.

VILLING et al. 2013-2015

Alexandra Villing, Marianne Bergeron, Giorgos Bourogiannis, Alan Johnston, François Leclère, Aurélia Masson, Ross Thomas, Naukratis: Greeks in Egypt, Londres, British Museum, 2013-2015, British Museum Online Research Catalogue, https://britishmuseum.org/collection/, consulté le 2 mai 2021.

\section{NOTES}

1. La mission d'étude, validée par les autorités du MoTA en mars dernier et initialement prévue en octobre et novembre, a été annulée.

2. La rédaction de plusieurs chapitres du tome II de la série, Les niveaux de la fin $d u v^{e}$ et $d u$ IV siècle av.J.-C., dévolu à l'habitat et aux sanctuaires contemporains du complexe palatial (publié en 2017), a pu être complétée.

3. DEFERNEZ, NOGARA, VALBELLE 2017.

4. Il s'agit de productions sans doute importées de la région memphite, d'après les récentes analyses conduites par Éric Goemaere, dans le cadre du programme AAP 17222 de l'Ifao (cf. rapport AAP 17222).

5. DEFERNEZ et al. 2020, p. 4-5.

6. Aussi qualifié de bol « achéménide ».

7. Ces espaces correspondent dorénavant aux unités 31, 44, 46, 96 et aux rues 31-33 (MARCHI 2014, p. 235).

8. Une baisse significative des échanges avec le monde grec, et en particulier avec la sphère athénienne, est sensible à Tell el-Herr, dès le début du IV $\mathrm{e}$. av. J.-C. - comme il est couramment attesté ailleurs.

9. Les espaces étudiés, E1-E2, E8 et E15, se situent sur le flanc nord-oriental du tell.

10. Voir les exemples illustrés dans DEFERNEZ et al. 2020, p. 6, fig. 3.

11. Voir les exemples récemment publiés dans DEFERNEZ, NOGARA, VALBELLE 2017, p. $162-166$.

12. Pour ce qui concerne le matériel grec de Naucratis, se référer en dernier lieu à la base de données en ligne mise en place par Alexandra Villing (viLLing et al. 2013-2015), sur le site du British Museum (https://britishmuseum.org/collection/, consulté le 
2 mai 2021). On consultera également la base de données de l'université d'oxford (www.beazley.ox.ac.uk, consulté le 2 mai 2021).

13. Dont de nouveaux exemples provenant des espaces 0153 (inv. P09/140) et SE26 (inv. P03/151).

14. LEHMANN et al. 2020.

15. DEFERNEZ et al. 2020, p. 4-5.

16. Prévu dans une étape ultérieure du programme.

17. Une mission d'étude est programmée par É. Goemaere, dans les locaux du pôle d'archéométrie de l'Ifao, en octobre 2021, à la suite de la table-ronde internationale organisée par les responsables du projet (cf. infra, rapport opération AAP 17122).

18. MARCHI 2014 ; DEFERNEZ, MARCHI, NOGARA 2017, p. 87-99.

19. DEFERNEZ et al. 2020, p. 7-9.

20. DEFERNEZ 2001.

21. Ces travaux ont récemment été valorisés par A. Banaszak, lors d'un colloque intitulé « Premières bouchées de pain », qui s'est tenu à Montpellier les 21-25 octobre 2019. Un poster présentait les différents témoignages connus dans la forteresse d'époque perse, en lien avec la fabrication du pain.

22. De nombreux fragments appartenant de toute évidence à la catégorie des fours ont été mis au jour durant les campagnes de fouilles des années 1980-1990: GRATIEN, SOUlí́ 1988, p. 25, fig. 1f ; LOUIS, GRATIEN 1990, p. 83, fig. 6d : 29 (sondage 3) ; DEFERNEZ 2001, pl. IV : 14b.

23. Se référer au précédent rapport de l'auteur dans DEFERNEZ et al. 2020, p. 8.

24. BANASZAK à paraître.

25. Ce type de procédé a déjà été mené, notamment avec une plaque à pain/plateaudokka issue du site de Qasr Allam (oasis de Bahariya), dont l'une des faces présente un médaillon central (MARCHAND 2017, p. 243, fig. 10f, g).

26. Hormis les moulages qui ne pourront s'effectuer que lors des missions d'étude, les essais proposés seront principalement menés en France, au parc archéologique d'Asnapio, à Villeneuve d'Ascq.

27. QAHERI à paraître.

28. QAHERI à paraître.

29. DEFERNEZ et al. 2020, p. 16-18 (rapport N. Favry).

30. FAVRY, MARCHI à paraître.

31. En charge de la documentation numérique des figurines de terre cuite (Patrimoine Archéologie).

32. DEFERNEZ et al. 2020, p. 19-20 (rapport P. Ballet).

33. Dans cette catégorie, signalons notamment les pièces suivantes : femmes debouts ou femmes couchées dans un édicule en forme de stèle, encadrée de colonnes surmontées d'un fronton; femmes debouts dans un petit édicule de type naos ou sur une simple plaquette d'argile, tenant un enfant, un récipient ou un animal (tel un capridé, un ibex, animal emblématique de l'art achéménide) et femmes drapées telles des Isis-Aphrodite. Quelques unes de ces pièces ont été récemment publiées dans un ouvrage de synthèse (BALLET 2020). Voir également les exemples publiés en ligne par Élodie Rotté (2012). 
34. Certaines pièces portaient encore des traces de polychromie.

35. On se référera en dernier lieu à l'ouvrage synthétique de l'auteure en charge de l'étude de cette documentation (BALLET 2020); plusieurs pièces recueillies sur le site en illustrent quelques propos.

36. DEFERNEZ et al. 2020, p. 13-14 (rapport S. Marchi).

INDEX

Thèmes : IFAO

Année de l'opération : 2020

sujets https://ark.frantiq.fr/ark:/26678/crtiUEzUHzs1A, https://ark.frantiq.fr/ark:/26678/

pcrtppJSngD0vR, https://ark.frantiq.fr/ark:/26678/pcrtFrREfvh9AM, https://ark.frantiq.fr/ark:/ 26678/pcrtbfqVEeganP, https://ark.frantiq.fr/ark:/26678/pcrtL7wUJowBSL

chronologie https://ark.frantiq.fr/ark:/26678/pcrtYHaws8Bjft, https://ark.frantiq.fr/ark:/

26678/pcrthWEseJqpny, https://ark.frantiq.fr/ark:/26678/pcrt3psX93pgp6

lieux https://ark.frantiq.fr/ark:/26678/pcrtFk68kkiDpk

nature https://ark.frantiq.fr/ark:/26678/crtPYLZpU4USm

\section{AUTEURS}

\section{CATHERINE DEFERNEZ}

CNRS, UMR 8167

NATHALIE FAVRY

Égyptologue, Sorbonne Université, UMR 8167

\section{ALINE BANASZAK}

Doctorante, université Lille 3, CNRS, UMR 8164 HALMA

\section{SÉPIDEH QAHERI}

Égyptologue, Collège de France 